PROCEEDINGS OF THE AMERICAN MATHEMATICAL SOCIETY

Volume 124, Number 1, January 1996

\title{
RESIDUALLY NILPOTENT ONE-RELATOR GROUPS WITH NONTRIVIAL CENTRE
}

\author{
JAMES McCARRON
}

(Communicated by Ronald Solomon)

\begin{abstract}
We determine explicitly the residually nilpotent one-relator groups with nontrivial centre. We show also that, if $G$ is a one-relator group, then $G$ is residually nilpotent if, and only if, its central quotient $G / Z(G)$ is residually nilpotent.
\end{abstract}

In this note we shall present an explicit determination of those one-relator groups with nontrivial centre that are residually nilpotent. The proof depends on the structure theory of one-relator groups with nontrivial centre developed by A. Pietrowski in $[\mathrm{P}]$.

In studying the residual nilpotence of groups which tend to be highly noncommutative, a concept which we call "compactness" is of some significance. A grouptheoretic class $\mathfrak{X}$ is termed compact if every noncyclic and residually nilpotent group in $\mathfrak{X}$ is residually a finite $p$-group, for some prime number $p$. The importance of this property results from the fact that the class of residually $p$-finite groups is much better behaved than the class of residually nilpotent groups. For example, a free product of residually $p$-finite groups is residually $p$-finite, while the free product of a group of order two and a group of order three is not residually nilpotent. Incidentally, this reflects the fact that the class of nontrivial free products of finite or cyclic groups is compact. Thus, if we wish to prove that a group is residually nilpotent, it is helpful to know that we may as well attempt to prove that it is residually a finite $p$-group, for some prime number $p$. In particular, the presence of torsion renders this approach exceedingly useful.

In $[\mathrm{M}]$, it was observed that the class of one-relator groups with torsion is compact. This raises the question whether the class of all one-relator groups is compact. As a first step towards dealing with torsion free one-relator groups, we sought to determine whether the class of one-relator groups with nontrivial centre is compact. Not only were we able to confirm this, but our investigation led to a complete and explicit determination of all residually nilpotent one-relator groups with nontrivial centre.

Here is our main result.

Received by the editors May 5, 1994.

1991 Mathematics Subject Classification. Primary 20E26, 20F05, 20F19.

Key words and phrases. Residually nilpotent, residually $p$-finite, one-relator group, compact.

The contents of this note form a part of the author's Ph.D. thesis. It is a pleasure to thank my supervisor, F. C. Y. Tang, for his help and guidance.

(C)1996 American Mathematical Society 
Main Theorem. Let $G$ be a one-relator group with nontrivial centre. Then $G$ is residually nilpotent if, and only if, one of the following obtains.

(a) $G$ is abelian;

(b) $G=\left\langle a, b \mid a^{\alpha}=b^{\beta}\right\rangle$, where $\alpha$ and $\beta$ are both powers of the same prime number; or

(c) $G=\left\langle a, b \mid\left[a, b^{\beta}\right]\right\rangle$, where $\beta$ is a power of a prime number.

Proof. That all the groups listed are residually nilpotent follows from [KM].

Let $G$ be a nonabelian, residually nilpotent one-relator group with nontrivial centre. Assume first that the abelianisation of $G$ is not free abelian of rank two. Then, by [P, Theorem 1], $G$ has a presentation of the form

$$
\left\langle a_{1}, a_{2}, \ldots, a_{m} \mid a_{1}^{\alpha_{1}}=a_{2}^{\beta_{1}}, a_{2}^{\alpha_{2}}=a_{3}^{\beta_{2}}, \ldots, a_{m-1}^{\alpha_{m-1}}=a_{m}^{\beta_{m-1}}\right\rangle,
$$

where $\alpha_{1}, \alpha_{2}, \ldots, \alpha_{m-1}$ and $\beta_{1}, \beta_{2}, \ldots, \beta_{m-1}$ are integers which satisfy

$$
\alpha_{i} \geq 2 \quad \text { and } \quad \beta_{i} \geq 2, \quad \text { for } 1 \leq i<m ;
$$

and

$$
\operatorname{gcd}\left\{\alpha_{i}, \beta_{j}\right\}=1, \quad \text { for } 1 \leq j<i<m .
$$

Let $A_{i}:=\left\langle a_{i} \mid \emptyset\right\rangle$, for $1 \leq i \leq m$. Then the presentation (1) reveals that $G$ is a tree product

$$
G=A_{1} \underset{a_{1}^{\alpha_{1}}=a_{2}^{\beta_{1}}}{*} A_{2}^{\underset{a_{2}^{\alpha_{2}}=a_{3}^{\beta_{2}}}{*}} \stackrel{\cdots}{\underset{a_{m-1}^{\alpha_{m-1}}=a_{m}^{\beta_{m-1}}}{*}} A_{m} .
$$

Therefore, the groups $G_{1}:=\left\langle a_{1}, a_{2}\right\rangle$ and $G_{2}:=\left\langle a_{2}, a_{3}\right\rangle$ have the one-relator presentations

$$
G_{1}=\left\langle a_{1}, a_{2} \mid a_{1}^{\alpha_{1}}=a_{2}^{\beta_{1}}\right\rangle \quad \text { and } \quad G_{2}=\left\langle a_{2}, a_{3} \mid a_{2}^{\alpha_{2}}=a_{3}^{\beta_{2}}\right\rangle .
$$

Since $G$ is residually nilpotent, hence, so are $G_{1}$ and $G_{2}$. By [M, Theorem 8.5] there are prime numbers $p_{1}$ and $p_{2}$ such that $\alpha_{1}$ and $\beta_{1}$ are powers of $p_{1}$, and such that $\alpha_{2}$ and $\beta_{2}$ are powers of $p_{2}$. From (3) we have $\operatorname{gcd}\left\{\alpha_{2}, \beta_{1}\right\}=1$, and hence, (2) implies that $p_{1} \neq p_{2}$.

Assume that $m \geq 3$. Let

$$
H:=\left\langle a_{1}, a_{2}, a_{3}\right\rangle=G_{1} \underset{\substack{\alpha_{2} \\ a_{2}=a_{3}^{\beta_{2}}}}{*} A_{3} .
$$

Since $G$ is residually nilpotent, therefore, $H$ is residually nilpotent.

Now, the element

$$
g:=\left[a_{1}^{\alpha_{2}}, a_{3}^{\beta_{1}}\right]
$$

is nontrivial. Thus, there is a prime number $q$ for which there is a normal subgroup $N$, of $q$-power index in $H$, such that $g$ does not belong to $N$. (This follows from a theorem of Gruenberg $[\mathrm{G}]$ which asserts that a finitely generated group is residually nilpotent if, and only if, it is residually of prime power order.)

Consider the finite $q$-group $\bar{H}=H / N$. If $q \neq p_{1}$, then there is an integer $k$ such that $\bar{a}_{1}=\bar{a}_{1}^{\alpha_{1} k}$. Then

$$
\bar{a}_{1}^{\alpha_{2}}=\bar{a}_{1}^{\alpha_{1} \alpha_{2} k}=\bar{a}_{2}^{\beta_{1} \alpha_{2} k}=\bar{a}_{3}^{\beta_{1} \beta_{2} k} \in Z_{\bar{G}}\left(\bar{a}_{3}^{\beta_{1}}\right) ;
$$

whence, $\bar{g}=\left[\bar{a}_{1}^{\alpha_{2}}, \bar{a}_{3}^{\beta_{1}}\right]=1$, a contradiction. Similarly, if $q \neq p_{2}$, we may deduce a contradiction. Since $p_{1}$ and $p_{2}$ are distinct, $q$ must be unequal to at least one of them, so we must, finally, reach a contradiction. Consequently, $m$ cannot be 
greater than or equal to three. It follows that $G=\left\langle a_{1}, a_{2} \mid a_{1}^{\alpha_{1}}=a_{2}^{\beta_{1}}\right\rangle$, where $\alpha_{1}$ and $\beta_{1}$ are powers of the same prime number $p_{1}$.

Now suppose that the abelianisation of $G$ is free abelian of rank two. By [P, Theorem 3], $G$ has a presentation of the form

$$
\left\langle t, a_{1}, a_{2}, \ldots, a_{m} \mid t^{-1} a_{1} t=a_{m}, a_{1}^{\alpha_{1}}=a_{2}^{\beta_{1}}, a_{2}^{\alpha_{2}}=a_{3}^{\beta_{2}}, \ldots, a_{m-1}^{\alpha_{m-1}}=a_{m}^{\beta_{m-1}}\right\rangle,
$$

where $\alpha_{1}, \alpha_{2}, \ldots, \alpha_{m-1}$ and $\beta_{1}, \beta_{2}, \ldots, \beta_{m-1}$ are integers satisfying (2) and (3) and the additional constraint that

$$
\alpha_{1} \alpha_{2} \cdots \alpha_{m-1}=\beta_{1} \beta_{2} \cdots \beta_{m-1} .
$$

This presentation exhibits $G$ as an HNN extension with base group having a presentation (1) in which (2) and (3) are satisfied. The residual nilpotence of $G$ implies the residual nilpotence of this base group. Consequently, from the first part of the proof, we must have $m=2$, and $G$ therefore has a presentation of the form

$$
G=\left\langle a, b, c \mid a^{-1} b a=c, b^{\beta}=c^{\beta}\right\rangle,
$$

where $\beta$ is a power of a prime number. (Here, we have changed our notation, thus: $b:=a_{1}, c:=a_{2}, \beta:=\alpha_{1}=\beta_{1}$, by (4).) To complete the proof, we merely apply Tietze transformations to this presentation for $G$ in order to eliminate $c$ :

$$
\begin{aligned}
G & =\left\langle a, b, c \mid c=a^{-1} b a, b^{\beta}=c^{\beta}\right\rangle \\
& =\left\langle a, b, c \mid c=a^{-1} b a, b^{\beta}=a^{-1} b^{\beta} a\right\rangle \\
& =\left\langle a, b \mid\left[a, b^{\beta}\right]\right\rangle .
\end{aligned}
$$

This completes the proof.

As a consequence of this, we obtain the following corollary, which was the original result sought.

Corollary 1 ("Compactness Theorem"). A noncyclic one-relator group with nontrivial centre is residually nilpotent if, and only if, it is residually a finite p-group, for some prime number $p$.

Proof. Let $G$ be a noncyclic and residually nilpotent one-relator group with nontrivial centre. If $G$ is abelian, then $G$ is free abelian of rank two, in which case $G$ is residually a finite $p$-group, for all prime numbers $p$. If $G$ is one among the groups in (b) of the Main Theorem, then $G$ is residually $p$-finite (where $\alpha$ and $\beta$ are powers of $p$ ), by [KM, Theorem 1.1]. Finally, if $G$ is one among the groups in (c) of the Main Theorem, then $G$ is residually a finite $p$-group (where $\beta$ is a power of $p$ ), by [KM, Theorem 3.2].

In view of the preceding corollary, we raise the following question.

Question. Is there a noncyclic and residually nilpotent one-relator group which is not residually a finite $p$-group, for any prime number $p$ ?

Such a group must therefore be torsion-free with trivial centre. In $[\mathrm{M}]$ it was shown that there is no example of the form $\left\langle a, b \mid a^{\alpha} b^{\beta} a^{\gamma} b^{\delta}\right\rangle$, where $\alpha, \beta, \gamma$ and $\delta$ are arbitrary integers.

From Pietrowski's solution of the isomorphism problem for one-relator groups with nontrivial centre $[\mathrm{P}]$, we may deduce the following consequence of the Main Theorem. 
Corollary 2. Given a one-relator group with nontrivial centre, there is an algorithm for determining whether or not it is residually nilpotent.

A group $G$ is nilpotent if, and only if, its central quotient $G / Z(G)$ is nilpotent. The statement obtained by replacing "nilpotent" by "residually nilpotent" is not generally valid. However, we showed in $[\mathrm{M}]$ that, if $\mathfrak{X}$ is any group-theoretic class and if $G$ is residually an $\mathfrak{X}$-group, then so is the central quotient $G / Z(G)$. Seen in this light, the following result therefore seems interesting.

Theorem 1. A one-relator group is residually nilpotent if, and only if, its central quotient is residually nilpotent.

Proof. Let $G$ be a (nonabelian) one-relator group with residually nilpotent central quotient $G / Z(G)$. Since the result is otherwise vacuous, we shall assume that the centre of $G$ is nontrivial. Consider, first, the case in which the abelianisation of $G$ is not free abelian of rank two. As before, $G$ then has a presentation (1) in which the conditions (2) and (3) are satisfied. By [P, Lemma 3], the centre of $G$ is given by

$$
Z(G)=\left\langle a_{1}^{\alpha_{1} \alpha_{2} \cdots \alpha_{m-1}}\right\rangle .
$$

Consequently, the central quotient $G / Z(G)$ of $G$ has the presentation

$$
\left\langle x_{1}, x_{2}, \ldots, x_{m} \mid x_{k}^{\alpha_{k}}=x_{k+1}^{\beta_{k}}(1 \leq k<m), x_{i}^{\beta_{1} \beta_{2} \cdots \beta_{i-1} \alpha_{i} \cdots \alpha_{m-1}}(1 \leq i \leq m)\right\rangle \text {, }
$$

where, for $1 \leq k \leq m$, the element $x_{k}$ is the image $a_{k} Z(G)$ of $a_{k}$ in $G / Z(G)$. The presentation (5) reveals $G / Z(G)$ as a tree product with finite cyclic vertex groups $\left\langle x_{1}\right\rangle,\left\langle x_{2}\right\rangle, \ldots,\left\langle x_{m}\right\rangle$. An argument almost identical to the one given in the proof of the Main Theorem shows that $m=2$. (We are assuming that $G / Z(G)$ is residually nilpotent. The only difference is that, here, the generators $x_{k}$ have finite order, but this does not affect the argument in any essential way.) Since $m=2$, therefore, $G$ has a presentation of the form $G=\left\langle a, b \mid a^{\alpha}=b^{\beta}\right\rangle$, where $\alpha$ and $\beta$ are both powers of some prime number $p$. Thus, $G$ is residually nilpotent, by the Main Theorem.

Now suppose that the abelianisation of $G$ is free abelian of rank two. Then as in the proof of the Main Theorem, $G$ has a presentation

$$
\left\langle t, a_{1}, a_{2}, \ldots, a_{m} \mid t^{-1} a_{1} t=a_{m}, a_{1}^{\alpha_{1}}=a_{2}^{\beta_{1}}, a_{2}^{\alpha_{2}}=a_{3}^{\beta_{2}}, \ldots, a_{m-1}^{\alpha_{m-1}}=a_{m}^{\beta_{m-1}}\right\rangle,
$$

where the integers $\alpha_{1}, \alpha_{2}, \ldots, \alpha_{m-1}$ and $\beta_{1}, \beta_{2}, \ldots, \beta_{m-1}$ satisfy (2), (3) and (4). From [P, Theorem 4] we have

$$
Z(G)=\left\langle a_{1}^{\alpha_{1} \alpha_{2} \cdots \alpha_{m-1}}\right\rangle .
$$

Consequently, $G / Z(G)$ has the presentation

$$
\begin{array}{r}
\left\langle y, x_{1}, x_{2}, \ldots, x_{m}\right| y^{-1} x_{1} y=x_{m}, x_{k}^{\alpha_{k}}=x_{k+1}^{\beta_{k}}(1 \leq k<m), \\
\left.x_{i}^{\beta_{1} \beta_{2} \cdots \beta_{i-1} \alpha_{i} \cdots \alpha_{m-1}}(1 \leq i \leq m)\right\rangle .
\end{array}
$$

Thus, $G / Z(G)$ is exhibited as an HNN extension, with stable letter $y$, and with base group having the presentation (5). We have just argued that a group defined by the presentation (5) must have $m=2$ if it is residually nilpotent. Thus, $G$ has the presentation

$$
G=\left\langle t, a, b \mid t^{-1} a t=b, a^{\beta}=b^{\beta}\right\rangle=\left\langle t, a \mid\left[t, a^{\beta}\right]\right\rangle .
$$

Now, $\beta$ must be a power of a prime number, so the Main Theorem implies that $G$ is residually nilpotent.

This completes the proof. 
We should draw attention to the following variant of this theorem.

Theorem 2. For any prime number $p$, a one-relator group is residually a finite p-group if, and only if, its central quotient is residually a finite p-group.

\section{REFERENCES}

[M] James McCarron, Residual nilpotence and one relator groups, Ph.D. Thesis, University of Waterloo, 1995, in preparation.

[G] K. W. Gruenberg, Residual properties of infinite soluble groups, Proc. London Math. Soc. (3) 7 (1957), 29-62. MR 19:386a

[P] Alfred Pietrowski, The isomorphism problem for one-relator groups with non-trivial centre, Math. Z. 136 (1974), 95-106. MR 50:2344

[KM] Goansu Kim and James McCarron, On residually p-finite one-relator groups, J. Algebra 169 (1994), 817-826. CMP 95:03

Department of Pure Mathematics, University of Waterloo, 200 University Avenue West, Waterloo, Ontario, Canada N2L 3G1

E-mail address: jmccarron@jeeves.uwaterloo.ca 